\title{
BORSUK'S ANTIPODAL AND FIXED-POINT THEOREMS FOR SET-VALUED MAPS
}

\author{
SHIOW-YU CHANG
}

(Communicated by James West)

\begin{abstract}
The purpose of this paper is to obtain the extensions of Borsuk's antipodal and fixed-point theorems for set-valued maps.
\end{abstract}

Borsuk [3] related the following relative results:

Borsuk's antipodal theorem. A single-valued antipodal-preserving continuous map $f: S^{n} \rightarrow E^{n}$ has a zero value.

Borsuk's fixed-point theorem. Let $U$ be a bounded symmetric convex open neighborhood of the origin in $E^{n}$, and let $f: \bar{U} \rightarrow E^{n}$ be antipodal-preserving on $\partial U$, i.e., $-f(a)=f(-a)$ for each $a \in \partial U$. Then $f$ has a fixed point.

In this note we consider the set-valued maps. With a proper definition of antipodality, we prove that Borsuk's antipodal theorem can be extended for setvalued maps on the boundary of a symmetric bounded balanced neighborhood of the origin. Such boundaries are more general than the homeomorphic image of spheres. At the same time we also prove Borsuk's fixed-point theorem on a symmetric balanced set of a locally convex space. The main results are summarized in Theorems 4, 5, 6, and 7. Lemmas 1 and 2 are two approximation properties which play a crucial role in this paper.

Let us first recall some definitions. Let $X$ and $Y$ be two topological spaces; capital letters $F: X \rightarrow Y$ denote nonempty set-valued maps while noncapital letters $f: X \rightarrow Y$ will denote single-valued functions. $F$ is called upper semicontinuous (u.s.c.) if for each open set $G$ of $Y$ the set $\{x \in X \mid F(x) \subset G\}$ is open in $X . F$ is said to have open lower sections if, for each $y \in Y$, $F^{-1}(y)=\{x \in X \mid y \in F(x)\}$ is open in $X$. The set $\operatorname{Gr} F$ is the graph of $F$ which is the set $\{(x, y) \mid y \in F(x), x \in X\}$. When $Y$ is a topological vector space and $S \subset Y$, $\cos$ denotes the convex hull of $S$. A set $B \subset Y$ is said to be balanced if $r B \subset B$ for every real number $r$ with $|r| \leq 1$.

Let $E$ be the normed space of all those sequences $x=\left(x_{1}, x_{2}, \ldots\right)$ of real numbers having at most finitely many $x_{n} \neq 0$, with the norm $\|x\|=\sum\left|x_{i}\right|$. The subset $\left\{x \in E \mid x_{i}=0\right.$ for all $\left.i>n\right\}$ is denoted by $E^{n}$; the unit $n$-sphere $S^{n}=\left\{x \in E^{n+1} \mid\|x\|=1\right\}$. Let $X$ and $Y$ be subsets of $E, S \subset X$ be a

Received by the editors February 14, 1991 and, in revised form, August 3, 1992.

1991 Mathematics Subject Classification. Primary 54H25, 55M20, 47H10.

Key words and phrases. Set-valued map, balanced set, antipodal map, fixed point. 
subset which is symmetric with respect to the origin, and $F: X \rightarrow Y$. We say that $F$ is antipodal on $S$ if $F(x) \cap(-F(-x)) \neq \varnothing$ for all $x \in S$. This is a generalization of the original single-valued functions to set-valued maps.

The following lemma is a generalization of a theorem of Cellina (see [1, Lemma 13.1, p. 67]). For different versions of the statement, we refer to [2, Theorem 1, p. 19].

Lemma 1. Let $X$ be a compact set and $\mathscr{B}_{1}$ an open symmetric neighborhood base at 0 in a topological vector space $E_{1}$. Let $Y$ be a set and $\mathscr{B}_{2}$ an open convex neighborhood base at 0 in a locally convex space $E_{2}$. Suppose the correspondence $F: X \rightarrow Y$ is u.s.c. with convex values. For $V \in \mathscr{B}_{1}$ define $F^{V}: X+V \rightarrow E_{2}$ via $F^{V}(x)=\operatorname{co\bigcup }_{z \in(x+V) \cap X} F(z)$. Then for each $W_{1} \in \mathscr{B}_{1}$ and $W_{2} \in \mathscr{B}_{2}$ there exists $V \in \mathscr{B}_{1}$ such that

$$
\mathrm{Gr} F^{V} \subset \mathrm{Gr} F+W_{1} \times W_{2}
$$

and $F^{V}$ has open lower sections.

Furthermore, if $S \subset X$ is symmetric with the origin and $F$ is antipodal on $S$, then $F^{V}$ is antipodal on $S+V$.

Proof. We choose $V_{1} \in \mathscr{B}_{1}$ such that $V_{1}+V_{1}+V_{1} \subset W_{1}$. For any $x \in X$ there exists $V_{x} \in \mathscr{B}_{1}$ such that $\bigcup_{z \in x+V_{x}} F(z) \subset F(x)+W_{2}$. We choose $U_{x} \in \mathscr{B}_{1}$ such that $U_{x}+U_{x}+U_{x} \subset V_{x}$.

Since $X$ is compact, there exists a finite cover $x_{1}+\left(U_{x_{1}} \cap V_{1}\right), \ldots, x_{n}+$ $\left(U_{x_{n}} \cap V_{1}\right)$ of $X$. Let $V=\left(\bigcap_{i=1}^{n} U_{x_{i}}\right) \cap V_{1}$. Fix $x \in X+V$. Then $x \in x_{i}+$ $\left(U_{x_{i}} \cap V_{1}\right)+V$ for some $i$. Hence $x+V \subset x_{i}+\left(U_{x_{i}} \cap V_{1}\right)+V+V \subset x_{i}+$ $\left(V_{x_{i}} \cap W_{1}\right)$. Thus $\bigcup_{z \in(x+V) \cap X} F(z) \subset \bigcup_{z \in\left(x_{i}+V_{x_{i}}\right)} F(z) \subset F\left(x_{i}\right)+W_{2}$. Since $F$ has convex values and $W_{2}$ is convex, $\operatorname{co\bigcup }_{z \in(x+V) \cap X} F(z) \subset F\left(x_{i}\right)+W_{2}$, and then $\{x\} \times F^{V}(x) \subset\left(\left\{x_{i}\right\}+W_{1}\right) \times\left(F\left(x_{i}\right)+W_{2}\right)$ for such $i$. Thus $\operatorname{Gr} F^{V} \subset$ $\mathrm{Gr} F+W_{1} \times W_{2}$.

Let $\mathrm{Gr} H=\mathrm{Gr} F+V \times\{0\}$. Then $H: X+V \rightarrow E_{2}$ has open lower sections obviously, and hence co $H: X+V \rightarrow E_{2}$ has open lower sections where $(\operatorname{co} H)(x)=\operatorname{co}(H(x))$ for each $x \in X+V$. For each $x \in X+V$, $\operatorname{co}(H(x))=\operatorname{co\bigcup }_{z \in(x+V) \cap X} F(z)=F^{V}(x)$. This implies that $F^{V}$ has open lower sections.

For any $x \in S+V$ there exist $x^{\prime} \in S, v \in V$ such that $x=x^{\prime}+v$. Since $S$ and $V$ are symmetric with the origin, $-x=-x^{\prime}+(-v) \in(S+V)$. Since $F$ is antipodal on $S, \varnothing \neq F\left(x^{\prime}\right) \cap\left(-F\left(-x^{\prime}\right)\right) \subset F^{V}(x) \cap\left(-F^{V}(-x)\right)$. Hence $F^{V}$ is antipodal on $S+V$. This completes the proof.

Lemma 2. Let $U$ be an open symmetric balanced neighborhood of the origin in $E^{n+1}$. Define $g: U \backslash\{0\} \rightarrow E^{n+1}$ by $g(x)=d(x, \partial U) x /\|x\|+x$. Then $g$ is continuous and symmetric; i.e., $g(-x)=-g(x)$. Furthermore, for any $\varepsilon>0$ there exists $k$ such that

$$
d\left(g^{k}(U \backslash\{0\}), \partial U\right)=\sup \left\{d\left(g^{k}(x), \partial U\right) \mid x \in U \backslash\{0\}\right\}<\varepsilon .
$$

Proof. By the property of $U, d(x, \partial U)$ is continuous and $d(x, \partial U)=$ $d(-x, \partial U)$ in $U \backslash\{0\}$. Thus $g$ is continuous, and $g(-x)=-g(x)$ for all $x \in U \backslash\{0\}$; i.e., $g$ is symmetric.

Let $r_{1}=\inf \{\|x\| \mid x \in \partial U\}$ and $r_{2}=\sup \{\|x\| \mid x \in \partial U\}$. For every $x \in$ $U \backslash\{0\}$ let $x_{I}=r_{1} x /\|x\|, x_{T}=r_{x} x$, where $r_{x}=\inf \{r \mid r x /\|x\| \in \partial U\}$. Then 
$r_{1} \leq\|g(x)\|=d(x, \partial U)+\|x\| \leq r_{x}$, and hence $g(x)=\|g(x)\| x /\|x\| \in$ $\overline{x_{I} x_{T}}$, where $\overline{x_{I} x_{T}}$ denotes the line segment from $x_{I}$ to $x_{T}$. Since $g^{j}(x)=$ $d\left(g^{j-1}(x), \partial U\right) x /\|x\|+\left\|g^{j-1}(x)\right\| x /\|x\|$, it follows that $\left\|g^{j-1}(x)\right\| \leq\left\|g^{j}(x)\right\|$ $=d\left(g^{j-1}(x), \partial U\right)+\left\|g^{j-1}(x)\right\| \leq r_{g^{j-1}(x)}=r_{x}$ and $g^{j}(x) \in \overline{g^{j-1}(x) x_{T}} \subset \overline{x_{I} x_{T}}$ for each $j=2,3, \ldots$.

Fix $x \in U \backslash\{0\}$, where $\|x\| \geq r_{1}$. If $d(x, \partial U)=\delta$, then there is $p_{x} \in \partial U$ such that $\left\|x-p_{x}\right\|=\delta$. If $p_{x}=x_{T}$, then for each $x^{\prime} \in \overline{x x_{T}}, d\left(x^{\prime}, \partial U\right)<$ $\delta$. If $p_{x} \neq x_{T}$, then each $x^{\prime} \in \overline{x x_{T}}$, the line $l$ passing through $x^{\prime}$ and parallel to $\overline{x p_{x}}$, intersects $\overline{o p_{x}}$ at $p_{x^{\prime}}$. Then $p_{x^{\prime}} \notin U$ and $d\left(x^{\prime}, \partial U\right) \leq$ $\left\|x^{\prime}-p_{x^{\prime}}\right\|=\left\|x^{\prime}\right\| \delta /\|x\| \leq r_{2} \delta / r_{1}$. Hence, if $\|x\| \geq r_{1}$ and $d(x, \partial U)<r_{1} \varepsilon / r_{2}$, then $d\left(\overline{x x_{T}}, \partial U\right)<\varepsilon$. Then $d\left(g^{j}(x), \partial U\right)<\varepsilon$ for $j=0,1,2, \ldots$.

Fix $y \in U \backslash\{0\}$. Then $\|g(y)\| \geq r_{1}$, and

$$
\begin{aligned}
g^{k}(y) & =d\left(g^{k-1}(y), \partial U\right) y /\|y\|+g^{k-1}(y) \\
& =\left[d\left(g^{k-1}(y), \partial U\right)+d\left(g^{k-2}(y), \partial U\right)+\cdots+d(g(y), \partial U)\right] y /\|y\|+g(y) \\
& =\left[d\left(g^{k-1}(y), \partial U\right)+\cdots+d(g(y), \partial U)+\|g(y)\|\right] y /\|y\| .
\end{aligned}
$$

Since

$$
\left\|g^{k}(y)\right\|=d\left(g^{k-1}(y), \partial U\right)+\cdots+d(g(y), \partial U)+\|g(y)\| \leq r_{2}
$$

and $\|g(y)\| \geq r_{1}, d\left(g^{k-1}(y), \partial U\right)+\cdots+d(g(y), \partial U) \leq r_{2}-r_{1}$, and hence there is some $i$ where $1 \leq i \leq k-1$ such that $d\left(g^{i}(y), \partial U\right) \leq\left(r_{2}-r_{1}\right) /(k-1)$. If $k>\left(r_{2}-r_{1}\right) r_{2} / r_{1} \varepsilon+2$, then $d\left(g^{i}(y), \partial U\right)<\left(r_{2}-r_{1}\right) r_{1} \varepsilon /\left[\left(r_{2}-r_{1}\right) r_{2}\right]=r_{1} \varepsilon / r_{2}<\varepsilon$, and hence

$$
d\left(g^{k}(y), \partial U\right)=d\left(g^{k-i}\left(g^{i}(y)\right), \partial U\right)<\varepsilon .
$$

This completes the proof.

Lemma 3. Let $E_{1}$ and $E_{2}$ be two topological vector spaces. Let $S \subset E_{1}$ and $S$ be compact and symmetric with respect to 0 . Let $F: S \rightarrow E_{2}$ be antipodalpreserving, convex-valued, and with open lower sections. Then $F$ has a singlevalued continuous antipodal selection $f: S \rightarrow E_{2}$.

Proof. By definition, $\left\{F^{-1}(y) \cap\left[-F^{-1}(-y)\right] \mid y \in E_{2}\right\}$ is an open cover of $S$; hence, there is a finite subcover $\Gamma=\left\{F^{-1}\left(y_{i}\right) \cap\left[-F^{-1}\left(-y_{i}\right)\right] \mid i=1,2, \ldots, n\right\}$. Let $\left\{\varphi_{i}\right\}_{i=1}^{n}$ be a partition of unity of $S$ with respect to $\Gamma$. It implies that each $\varphi_{i}$ is nonnegative continuous on $S, \sum \varphi_{i}(x)=1$, and $\varphi_{i}(x)>0 \Rightarrow x \in$ $F^{-1}\left(y_{i}\right) \cap\left[-F^{-1}\left(-y_{i}\right)\right]$. Define $p: S \rightarrow E_{2}$ by

$$
p(x)=\frac{\sum_{i=1}^{n} \varphi_{i}(x) y_{i}-\sum_{i=1}^{n} \varphi_{i}(-x) y_{i}}{\sum_{i=1}^{n} \varphi_{i}(x)+\sum_{i=1}^{n} \varphi_{i}(-x)} .
$$

Then $p$ is continuous, and $-p(-x)=p(x)$ for all $x \in S$. If $\varphi_{i}(x) \neq$ 0 , then $x \in F^{-1}\left(y_{i}\right)$, and hence $y_{i} \in F(x)$. If $\varphi_{i}(-x) \neq 0$, then $-x \in$ $-F^{-1}\left(-y_{i}\right)$, and hence $-y_{i} \in F(x)$. Since $F$ is convex-valued, $p(x) \in F(x)$ for all $x \in S$.

Borsuk antipodal and Borsuk-Ulam theorems [3, $\S 4$, Theorem 5.2, p. 44] can be generalized to the following two theorems.

Theorem 4. Let $U$ be an open bounded symmetric neighborhood of the origin in $E^{n+1}$, and let $F: \partial U \rightarrow E^{n}$ be u.s.c., closed, convex-valued, and antipodalpreserving. Then $F$ has a zero. 
Proof. Let 0 denote the zero map from $\partial U$ into $\{0\}$. Suppose that $F$ does not have zero. Then by [4, Theorem 1] there are open convex neighborhoods $W$ of 0 in $E^{n}$ and $W_{1}$ of 0 in $E^{n+1}$ such that $\left[\mathrm{Gr} F+W_{1} \times W\right] \cap \mathrm{GrO}=$ $\varnothing$. By Lemma 1 there is an open neighborhood $V$ of 0 in $E^{n+1}$ such that $\mathrm{Gr} F^{V} \subset \mathrm{Gr} F+W_{1} \times W$ and the domain of $F^{V}$ is $\partial U+V$. Let $V_{1}$ be a closed bounded symmetric balanced neighborhood of 0 in $E^{n+1}$ such that $V_{1}+V_{1} \subset V$ and $\left.F^{V}\right|_{\partial U+V_{1}}$ denote the restriction of $F^{V}$ on $\partial U+V_{1}$. Choose $\lambda, 0<\lambda<1$, such that $\lambda S_{n} \subset U$. Define $g$ on $U \backslash\{0\}$ as Lemma 2. Then there exists a positive integer $k$ such that $g^{k}(x) \in \partial U+V_{1}$ for all $x \in \lambda S_{n}$. Let $K=g^{k}\left(\lambda S_{n}\right)$. By Lemma $1,\left.F^{V}\right|_{\partial U+V_{1}}$ is antipodal and has open lower sections. By Lemma 3, $\left.F^{V}\right|_{K}$ has a single-valued continuous antipodal selection $h$. Define $f: S_{n} \rightarrow E^{n}$ by $f(x)=h\left(g^{k}(\lambda x)\right)$. Then $f$ is a single-valued continuous antipodal map with zero free. This is a contradiction to Borsuk's antipodal theorem. This completes the proof.

Theorem 5. Let $U$ be an open bounded symmetric neighborhood of the origin in $E^{n+1}$. Then every u.s.c. closed convex-valued map $F: \partial U \rightarrow E^{n}$ has an intersection point for at least one pair of antipodal points.

Proof. Define $G: \partial U \rightarrow E^{n}$ by $G(x)=F(x)-F(-x)$. Then $G$ is a u.s.c. closed convex-valued antipodal-preserving map. By Theorem 4, $0 \in F(x)-$ $F(-x)$, and hence $F(x) \cap F(-x) \neq \varnothing$ for some $x \in \partial U$. This completes the proof.

Theorem 6. Let $U$ be an open bounded symmetric balanced neighborhood of the origin in $E^{n+1}$. Let $F: \bar{U} \rightarrow E^{n+1}$ be u.s.c., closed, convex-valued, and antipodal on $\partial U$; i.e., $F(a) \cap[-F(-a)] \neq \varnothing$ for each $a \in \partial U$. Then $F$ has $a$ zero value and a fixed point on $\bar{U}$.

Proof. Suppose that $F$ has no zero value. Let $\mathbf{0}$ denote the zero map from $\bar{U}$ into $\{0\}$. Then $\operatorname{Gr} F \cap \operatorname{Gr} 0=\varnothing$. By [4, Theorem 1] there is a closed convex neighborhood $W$ of 0 in $E^{n+1}$ such that $[\mathrm{Gr} F+W \times W] \cap[\mathrm{Gr} 0+W \times W]=$ $\varnothing$. By Lemma 1 there exists a symmetric neighborhood $V$ of 0 such that $\operatorname{Gr}\left(F^{V}\right) \subset \operatorname{Gr} F+W \times W$. Hence $0 \notin F^{V}(x)$ for all $x \in \bar{U}+V$. By Lemma $3,\left.F^{V}\right|_{\bar{U}}$ has a single-valued selection $f$ such that $f$ is antipodal on $\partial U$.

Define $J: \bar{U} \rightarrow E^{n+2}$ by $J(x)=x+d(x, \partial U) \vec{e}_{n+2}$. Then $J$ is one-toone continuous, and $J(x)=x$ on $\partial U$. Let $V=\left\{x+u \vec{e}_{n+2}|x \in U| u \mid,<\right.$ $d(x, \partial U)\}$. Then $V$ is open, bounded, symmetric, and balanced in $E^{n+2}$ and $\partial V=J(\bar{U}) \cup\{-J(\bar{U})\}$.

Define $H: \partial V \rightarrow E^{n+1}$ by

$$
H(x)= \begin{cases}f\left(J^{-1}(x)\right) & \text { if } x \in J(\bar{U}), \\ -f\left(J^{-1}(-x)\right) & \text { if } x \in-J(\bar{U}) .\end{cases}
$$

Then $H$ is a continuous antipodal single-valued function with zero free. This is a contradiction to Theorem 4 . Thus $F$ has a zero value.

By the above conclusion, $F-I$ has a zero value; i.e., there exists $x \in \bar{U}$ such that $0 \in F(x)-\{x\}$. Hence $x \in F(x)$ for some $x \in \bar{U}$. This proves the theorem.

The following theorem is a generalization of a result of Borsuk $[3, \S 4$, Theorem 3.3 , p. 57]. 
Theorem 7. Let $M$ be a closed bounded symmetric balance set at 0 in a locally convex space $E$. Let $F: M \rightarrow E$ be a u.s.c. closed convex-valued map such that the closure of $F(M)$ is compact and $F$ is antipodal on the boundary of $M$. Then $F$ has at least one fixed point.

Proof. Let $\mathscr{B}$ denote a closed bounded symmetric convex neighborhood base at 0 in $E$. For each $V \in \mathscr{B}$ there is a finite subset $S$ of $F(M)$ such that $(y+V) \cap S \neq \varnothing$ for each $y \in F(M)$. Let $S_{V}$ be a finite subset of $E$ such that $S \subset S_{V}$. Let $H_{S_{V}}$ denote the finite-dimensional space spanned by $S_{V}$.

Define $G_{V}: M \cap H_{S_{V}} \rightarrow H_{S_{V}}$ by $G_{V}(x)=(F(x)+V) \cap H_{S_{V}}$. Then $G_{V}$ is u.s.c. with nonempty compact convex values. Since $F(a) \cap-F(-a) \neq \varnothing$ for all $a \in \partial\left(M \cap H_{S_{V}}\right), \varnothing \neq\{[F(a) \cap(-F(-a))]+V\} \cap H_{S_{V}}$ for all $a \in \partial\left(M \cap H_{S_{V}}\right)$; i.e., $G_{V}$ is antipodal on its boundary. If 0 is a relative interior point of $M \cap H_{S_{V}}$, then by Theorem 6 there is $x_{V}$ such that $x_{V} \in G_{V}\left(x_{V}\right)$; i.e., $x_{V} \in F\left(x_{V}\right)+V$. On the other hand, if $0 \in \partial\left(M \cap H_{S_{V}}\right)$, then $M \cap H_{S_{V}}=\partial\left(M \cap H_{S_{V}}\right)$. Since $G_{V}$ is antipodal on $M \cap H_{S_{V}}$, we have $G_{V}(0)=-G_{V}(0)$. Since $G_{V}(0)$ is convex, we have $0 \in G_{V}(0)$.

From the above argument, we get that in any situation for each $V \in \mathscr{B}$ there exist $x_{V} \in M$ such that $x_{V}=y_{V}+v$, where $y_{V} \in F\left(x_{V}\right)$ and $v \in V$. Since the closure of $F(M)$ is compact, there is a subnet of $\left\{y_{V}\right\}$ that converges to $x_{0}$. Then $x_{0} \in F\left(x_{0}\right)$. This completes the proof.

Now we give the following application of Theorem 4 which is a result of Krein, Krasnoselsky, and Milman [3, $\S 5$, Theorem 5.4, p. 80].

Theorem 8. Let $M, N$ be linear subspaces of a Banach space $(E,\|\|)$. If $\operatorname{dim} M>\operatorname{dim} N$, then there is a $x_{0} \in M$ such that $d\left(x_{0}, N\right)=\left\|x_{0}\right\|>0$.

Proof. For each $x \in E$ let $F(x)=\{y \in N \mid d(y, x)=d(x, N)\}$. Then $F(x)$ is compact convex and $x \rightarrow F(x)$ is of closed graph. Furthermore, the map $F: E \rightarrow N$ has the property $F(-x)=-F(x)$ for all $x \in E$. Let $S_{M}$ denote the unit sphere in $M$. Consequently, applying Theorem 4 to $\left.F\right|_{S_{M}}: S_{M} \rightarrow N$, we obtain a point $x_{0} \in S_{M}$ such that $0 \in F\left(x_{0}\right)$. Clearly, $x_{0}$ is the required point. This proves the theorem.

\section{REFERENCES}

1. Kim C. Border, Fixed point theorems with applications to economics and game theory, Cambridge Univ. Press, London and New York, 1985.

2. A. Cellina, Approximation of set-valued functions and fixed point theorems, Ann. Mat. Pura Appl. (4) 4 (1969), 17-24.

3. J. Dugundji and A. Granas, A fuxed point theory, Monograf. Mat., vol. 61, PWN, Warsawa, 1982.

4. Shiow-Yu Chang, Separation and von Neumann intersection theorems, Proc. Amer. Math. Soc. 112 (1991), 1149-1152.

Department of Mathematics, Soochow University, Taipei, Taiwan, Republic of China 